\title{
PAPER
}

\section{Spectrum analysis and tone quality evaluation of piano sounds with hard and soft touches}

\author{
Hideo Suzuki \\ Department of Information and Network Science, Chiba Institute of Technology, \\ 2-17-1 Tsudanuma, Narashino, 275-0016 Japan
}

(Received 16 March 2006, Accepted for publication 16 May 2006)

\begin{abstract}
Whether the piano touch can affect the piano tone quality or not has been a subject of discussion for more than 70 years. Many studies have been conducted on this subject, but most of the conclusions so far were on the negative side. This is possibly because the effect of the touch on physical and tone quality differences are so small and, therefore, very careful recording and analysis are needed to find the subtle differences. This paper describes results of spectrum analysis of hard and soft touch tones, which were recorded with a maximum attention on the equality of sound levels of tones to be compared. Recording and analysis of notes G3, G4, and G5 showed that the touch can affect the spectrum of sound only for G5 in a very small degree, which might become invisible without careful graphical comparisons. The listening tests conducted using recorded sounds showed that not all but some people can discriminate the differences of tone quality between the hard and sort touches. The result of this study is very important since it showed, for the first time, that the touch can produce physical and psychological differences of piano tones even though in a much lesser degree than most pianists expect.
\end{abstract}

Keywords: Touch, Tone quality, Spectrum analysis

PACS number: 43.75.-z, 43.75.Mn [doi:10.1250/ast.28.1]

\section{INTRODUCTION}

Whether the piano touch can affect the piano tone quality or not has been a subject of argument for a long time [1-3]. The touch here is defined, in a narrow sense, as a way a person (or more specifically, a pianist) presses a key to produce a single piano tone with an intention to add some character to the tone quality. Since the piano tone has a nonlinear characteristic (relative levels of higher order partials increase as the intensity of the piano tone increases), one needs to compare piano tones with different touches while keeping the peak levels constant or at least within a negligible difference. Pianists in general think that they can change the tone quality by the touch. On the other hand, physics-oriented scientists tend to think that there is no way to control the interaction between the hammer and the string except by the hammer speed. A reason for this is that the hammer travels with a constant speed (neglecting the effect of the gravity and a mechanical loss) after a release from the acceleration by the key. The latter assumes that the hammer comprises a pure mass and a spring. If this is the case, there is no way to control the tone quality if the sound level is kept constant. However, there is a possibility that the hammer shank may be bent even before the contact due to the acceleration by the key. Suzuki analyzed the effect of the initial shank deformation on the spectrum of the impact force of the hammer [4,5]. Experimental results obtained by Askenfelt and Jansson clearly show that the touch affects the motion of the hammer before the contact [6].

Koornhof and van der Walt studied the effect of touch (legato and staccato) on the tone quality of note G3. They found that the effect of the touch was a generation of early noises in the case of staccato touch [7]. Miura reported a study on the tone quality of note $\mathrm{C} 5$, with an emphasis on the key-keybed noise [8]. Naganuma and Nakamura investigated differences of the preceding, longitudinal, and transverse waves due to the touch, but the playing levels were not monitored and the number of data was too small to make definitive conclusions [9]. Goebl et al. made a study on the same subject and their conclusion was that the finger-key noise produced by the finger hitting the key was the only audible difference. There was no sound quality difference if the noise was removed [10].

Suzuki et al. compared the power spectrum distributions of notes G3, G4, and G5 played by "hard" and "soft" touches while keeping peak levels the same [11]. A meaningful spectrum difference caused by the difference of 
the touch was detected only for note G5. This result led to the next study on whether the amount of the spectrum difference generated by the touch was audible or not. This paper describes both results including those reported in reference [11] and results of recently conducted listening experiments.

\section{SPECTRUM AND WAVEFORM DIFFERENCES DUE TO THE TOUCH}

\subsection{Recording of Piano Tones with Hard and Soft Touches}

The "hard" touch is defined here as pressing the key while keeping the shoulder, elbow, wrist and finger as firm (tight) as possible. The "soft" touch is defined as its opposite. These two touches were chosen because the professional pianist Kanno thought that he could produce the largest tone quality difference by these touches. The notes chosen were G3, G4 and G5, which cover the main octaves of the piano scale.

A recording of piano tones took place at a privately owned studio with dimensions of $13 \mathrm{~m} \times 9.2 \mathrm{~m} \times 4.8 \mathrm{~m}$. Apparatus used for the recording are as follows.

Piano: Model D (Steinway \& Sons)

Microphone: CU-41 (Sanken Microphone)

Mixing amp: SX-PR (Sonosax)

Digital recorder: D1624mkII (Fostex)

Sound level meter: LA-5111 (Ono Sokki)

Microphone CU-41 is designed for professional use with its frequency range from $20 \mathrm{~Hz}$ to $30 \mathrm{kHz}$. Digital recorder D1624mkII has specifications of $96 \mathrm{kHz}$ sampling and 24 bit quantization, which were used for recording. The sound level meter played an important role in this experiment in order to keep the played sound levels within a tolerance range. Peak levels of individual tones were monitored by use of a sound level meter. The relative positioning of the piano and the microphones are shown in Fig. 1. The microphones were kept relatively far from the keys in order to lessen contaminations by the finger-key and key-keybed noises.

First, note G3 was played several times with the hard touch until its peak level fell within $\pm 0.3 \mathrm{~dB}$ of a target level ( $98 \mathrm{~dB}$ for $\mathrm{G} 3$ ). Then, the same note was played by the soft touch until the peak level fell within $\pm 0.3 \mathrm{~dB}$ of the previously recorded sound. This process was repeated 10 times, giving ten pairs of sound signals with a peak level difference within $\pm 0.3 \mathrm{~dB}$. The same recording was repeated for notes G4 and G5, with the target levels of $97 \mathrm{~dB}$ and $90 \mathrm{~dB}$, respectively. The damper pedal was not used in this recording.

It should be noted that the limits of the playing levels posed quite a challenge to the pianist to play notes with different touches within a required intensity range.

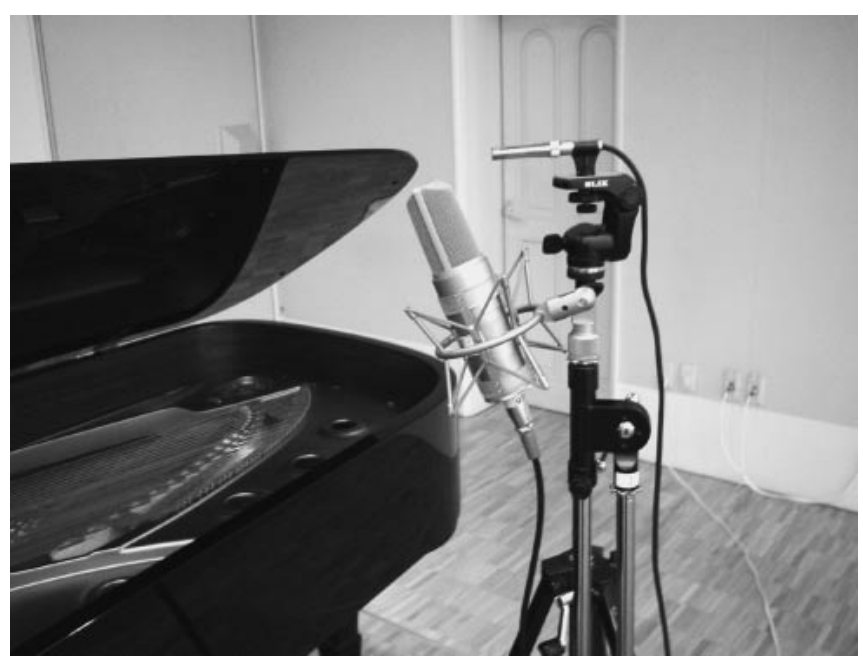

Fig. 1 Setting of a Steinway and Sons model D grand piano and microphones used for recording and peak level monitoring.

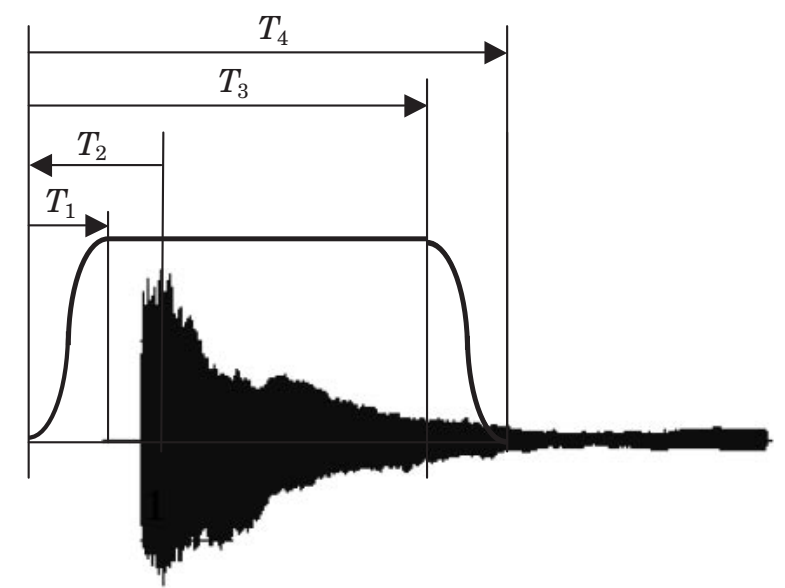

Fig. 2 Time window function used for editing the piano sound.

\subsection{Editing and Analysis of the Recorded Sounds}

Since each piano tone was recorded for 5 seconds or more, it must be edited before the spectrum analysis so that the edited signals have the same onset and duration. A time window $w(t)$ used for this purpose is shown in Fig. 2. The time window is defined as:

$$
\begin{aligned}
w(t) & =\sin ^{2}\left(\pi t / 2 T_{1}\right) & & 0 \leq t \leq T_{1} \\
& =1 & & T_{1} \leq t \leq T_{3} \\
& =\cos ^{2}\left[\pi\left(t-T_{3}\right) /\left\{2\left(T_{4}-T_{3}\right)\right\}\right] & & T_{3} \leq t \leq T_{4}
\end{aligned}
$$

At first, the time $\left(T_{2}\right)$, at which the absolute instantaneous sound pressure takes the maximum value, is found. Then, $T_{1}, T_{3}$, and $T_{4}$ are determined. The times $T_{1}-T_{4}$ that were used for G3, G4, and G5 are shown in Table 1. The waveform at the onset of the piano tone is not affected by the windowing. However, the tail of the waveform is gradually reduced to zero so that the duration of the signal 
Table 1 Time parameters used in the time window functions.

\begin{tabular}{ccccc}
\hline & T1 & T2 & T3 & T4 \\
\hline G3 & 0.04 & 0.16 & 2.52 & 2.731 \\
G4 & 0.04 & 0.16 & 2.52 & 2.731 \\
G5 & 0.04 & 0.1 & 2.23 & 2.731 \\
\hline
\end{tabular}

(including null before the onset) is equal to $2.73067 \mathrm{~s}$ $\left(=2^{18} / 96,000\right.$, where $2^{18}$ is the number of sampling points and 96,000 is the sampling frequency).

In order to compare the spectrum differences, the fast Fourier transform (FFT) was applied to individual signals $x(n)$ after editing. Then, a moving average was applied to spectrum data $X(k)$, giving the new power spectrum data:

$$
X^{\prime}(k)=\left[\sum_{i=-3}^{3}|X(k+i)|^{2}\right] / 7 \quad k \geq 3
$$

This averaging was applied in order to take care of the spectral spreading of each partial for an accurate comparison of spectrum differences due to the touch.

\subsection{Spectrum Differences}

Spectral differences of partials were investigated, since we were interested in physical qualities produced by the vibration of string(s) as distinguished from noises produced by other sound generation mechanisms. Peak levels that correspond to individual partials were extracted from spectrum data $X^{\prime}(k)$ given by Eq. (2). Figure 3 shows examples of spectrum levels of partials for G3, G4, and G5. Maximum levels of partials (2nd for G3 and 1st for G4 and G5) were all made equal to $100 \mathrm{~dB}$ for graphical comparisons. Accordingly, levels of other partials were also adjusted by the same amount. Above these highest orders (25, 17, and 10 for G3, G4, and G5, respectively), it becomes difficult to distinguish spectral peaks of partials

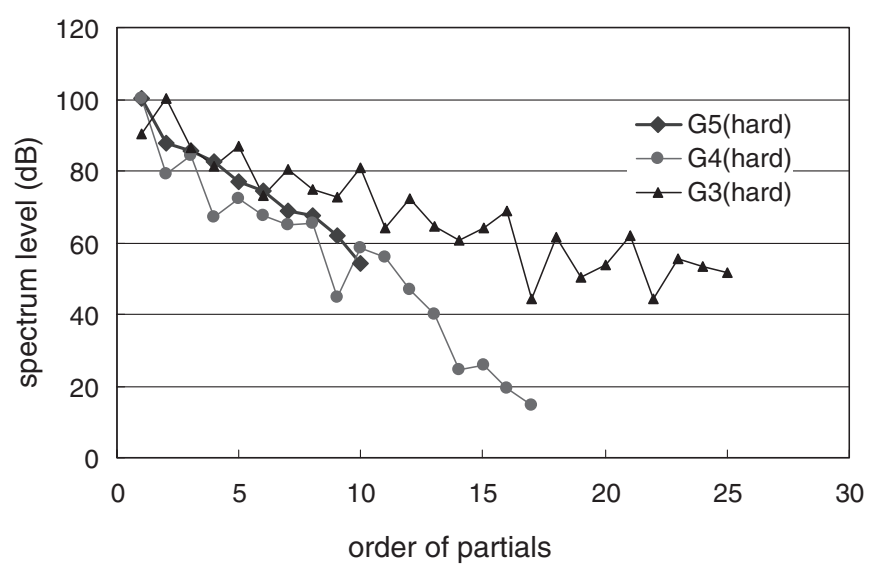

Fig. 3 Examples of relative spectrum levels of partials for G3, G4, and G5 tones.

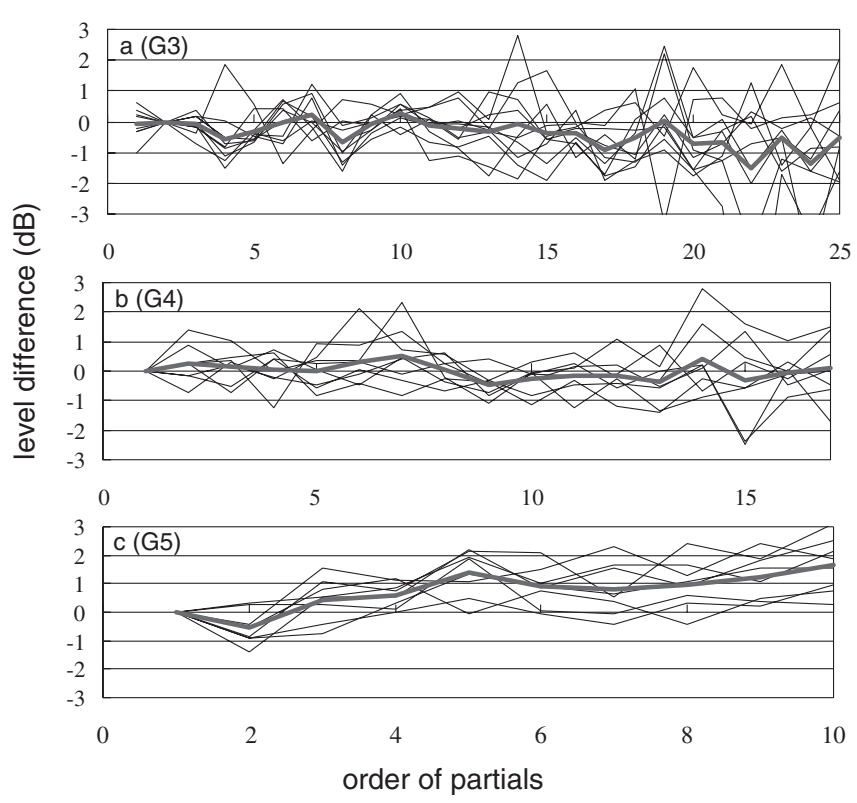

Fig. 4 Spectral level differences of sounds produced by hard- and soft-touches. Thin lines are for individual pairs of recording and the thick line is mean level differences.

from the noise floor. The top (a), middle (b), and bottom (c) charts of Fig. 4 show level differences of partials of hard and soft touch sounds for notes G3, G4, and G5, respectively. Thin lines are for individual pairs of recording (only 8 thin lines for G5 are shown because one pair was not correctly recorded and another pair was damaged during the processing). Positive values indicate that the hard touch has larger partial levels than the soft touch. At first glance, we see that the effect of the touch is negligible for G3 and G4. For these notes, the fluctuations of individual curves are much larger than the averaged values, which are close to zero. In the case of G5, however, we can observe a general trend of increase of the averaged level difference as the order of partial increases. The averaged curve has a $1.5 \mathrm{~dB}$ level difference at the 10th partial. If we look at the results of G3 more carefully, there is a slight decrease in level as the order becomes larger. There is a possibility that the effect of the shank deformation at the time of hammer-string contact is different with different key numbers. The shank deformation may be least effective in the case of G4 among three tones tested, and it may have opposite effects on G3 and G5.

Pianist Kanno commented during the recording that the touch works most effectively on the tone quality roughly in the range from $\mathrm{G} 3$ to $\mathrm{G} 4$, which is contrary to the spectrum differences found in this study. The effect of the touch, assuming its existence, is very inconsistent in this range as Fig. 4 shows. On the other hand, in the case of G5, the touch can produce spectrum differences even though they seem to be much smaller than most pianists expect. 


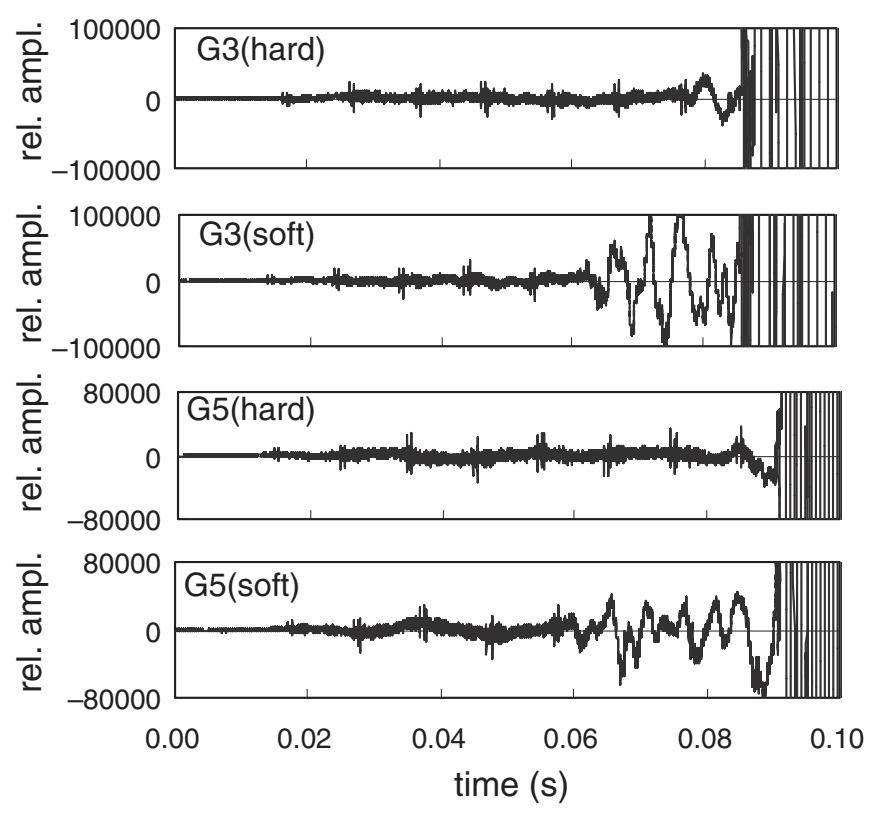

Fig. 5 Two functions used to modify the spectrum distribution of a piano tone.

\subsection{Preceding Tones/Noises}

Physical differences due to the touch found in the previous studies [7-10] were mostly those of tones or noises that precede the main component of the piano tone. The existence of differences of preceding tones and/or noises due to the touch was also checked in this study. An example of the zoomed-up waveforms of G3 (top two charts) and G5 (bottom two charts) for the hard and soft touches during the initial 0.1 second period is shown in Fig. 5. The zooming rate of the vertical axis is 100 (the maximum absolute value of the waveform is approximately $7 \times 10^{6}$ for G3 and $5 \times 10^{6}$ for G5). Figure 5 shows that there are two kinds of preceding tones or noises. The first one, which is common for the hard and soft touches, seems to be an ambient noise (possibly including the electrical noises of the recording system). The period of the spiky noises is 0.01 second, indicating that they are related to the frequency of the electric power $(50 \mathrm{~Hz}$ in Japan). The second one is mostly seen in the waveforms of the soft touch, which is probably generated by the impact of the key to the keybed (key-keybed noise). In playing the soft touch tones, it is necessary to push the key all the way to the end in order to produce the equal intensity of tones with the one played by the hard touch. In the hard touch, the key is mostly accelerated at the beginning and the key must be slowed down when it hits the keybed. The same discussion is given by Miura [8]. The preceding tones in Fig. 5 are very similar to those observed by Koornhof and van der Walt in the tones of staccato touch [7]. They explained that this type of noise is generated by a large acceleration of some parts of the key action mechanism.

Two important facts are found in Fig. 5. The first one is that the (second) preceding noise has a significantly lower frequency than the fundamental frequency of the following main component. The second one is that the hard touch sound, which has larger partial levels, has smaller preceding noise than the soft touch sound. Therefore it is reasonable to assume that the preceding noise has very small effect on the spectrum difference shown in Fig. 4 and consequently very small effect on the sound quality of G5 sounds.

\section{TONE QUALITY EVALUATION}

In section 2, it was shown that the hard and soft touches produce sounds with different spectrum distributions in case of G5. Then a question arises whether the difference is audible or not. In this section, results of two types of listening tests are reported.

\subsection{Difference Limen of Spectral Distribution Varia- tion}

The first listening test was conducted using a pair of sound (a) and sound (b), which are obtained by applying the frequency characteristics shown in Fig. 6 to a recorded (and edited) G5 piano sound. Curve (a) has a response defined by Eq. (3).

$A_{\mathrm{dB}}(f)=\left\{\begin{array}{ll}-c_{\mathrm{dB}} & 0 \leq f \leq f_{0} \\ (L / 8) \log _{2}\left(f / f_{0}\right)-c_{\mathrm{dB}} & f_{0} \leq f \leq 16 f_{0} \\ L / 2-c_{\mathrm{dB}} & 16 f_{0} \leq f\end{array}\right\}$

$A_{\mathrm{dB}}(f)$ is flat up to $786.6 \mathrm{~Hz}\left(=f_{0}\right)$, which is slightly above the nominal frequency of G5 $(784 \mathrm{~Hz})$, and then linearly increases with a gradient of $L / 8(\mathrm{~dB} /$ octave, where $L / 2$ is the level of the plateau) until the frequency reaches 4 octaves above $786.6 \mathrm{~Hz}$, then again becomes flat beyond this frequency. Curve (b) has the opposite characteristic. Values of $A(f)$, which is a linear version of $A_{\mathrm{dB}}(f)$, is multiplied to the complex spectrum of a recorded sound

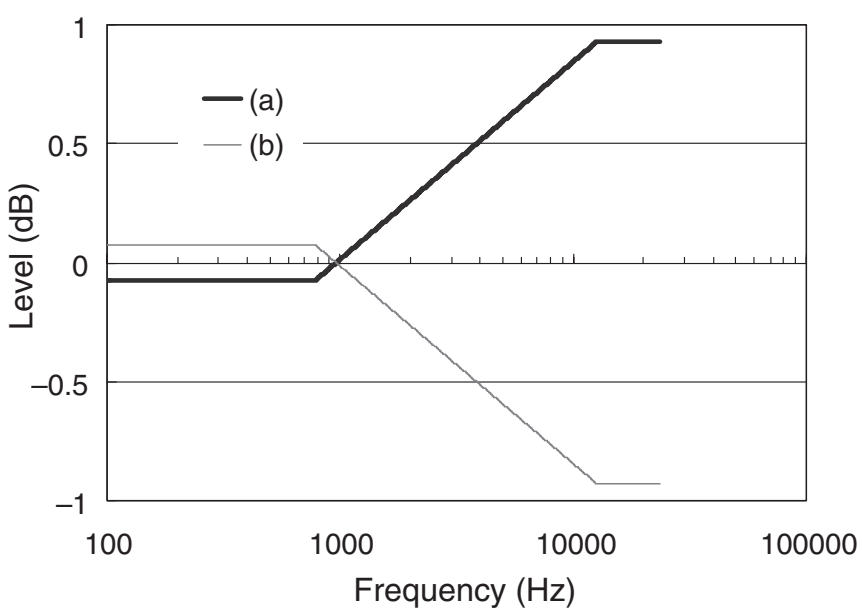

Fig. 6 Difference limens (dB) of nine subjects. 
chosen arbitrarily from 8 hard touch samples. The value $c_{\mathrm{dB}}$ was adjusted so that the sound (a) and sound (b) have the same energy, a time integration of the instantaneous power.

Ten senior students in the author's laboratory participated in the listening test. None of them had any specific musical training. After a number of practices, each of which lasted approximately 30 minutes, they took a preliminary test. In this preliminary test, test sample pairs with various $L s$ in $0.5 \mathrm{~dB}$ steps were presented. Twenty pairs of (a)-(b) and (b)-(a) combinations (ten each) were presented to each subject and a percentage of correct judgment was obtained. Each subject was allowed to listen to one pair as many times as he or she wanted before making a judgment. Each judgment is counted as correct if the subject judges sound (a) as the tone with higher frequency components i.e. obtained using the response (a) in Fig. 6. If the judgments were opposite but consistent, then it would have been considered that the subject could discriminate the sound quality difference, but this kind of situation never happened.

After the preliminary test, a formal listening test was conducted using pairs with various $L s$ in $0.05 \mathrm{~dB}$ steps. The smallest level difference $L$ of an (a) and (b) tone pair, that each subject judged correctly with a percentage of 80 or more, is shown as a difference limen for each subject (DL) in Fig. 7 (one subject was omitted because his result was found erroneous). The minimum, maximum, and average DLs are $1.25,3.3$, and $2.15 \mathrm{~dB}$ respectively. The ability to discern the sound quality due to the spectrum difference is significantly dependent on the individual subject. Five subjects out of nine have DLs that are lower than or equal to $1.8 \mathrm{~dB}$. Since $L=1.8 \mathrm{~dB}$ at the 16 th partial corresponds to a level difference of $1.5 \mathrm{~dB}$ at the 10 th partial, it is expected that several people out of ten may be able to hear the difference in tone quality between two sounds with the average spectrum difference shown in Fig. 4(c).

3.2. Direct Comparisons of the Recorded Sound Pairs

In the first sound quality evaluation test mentioned

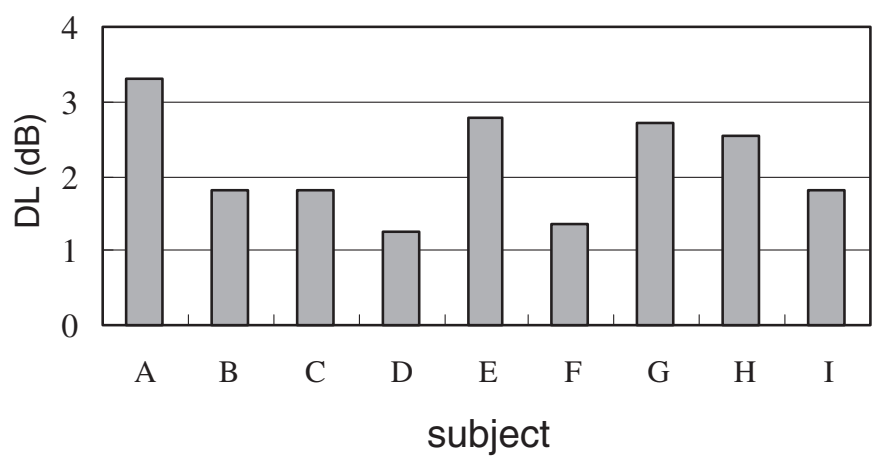

Fig. 7 Relative level differences of partials for pairs \#1, \#2, and \#3.

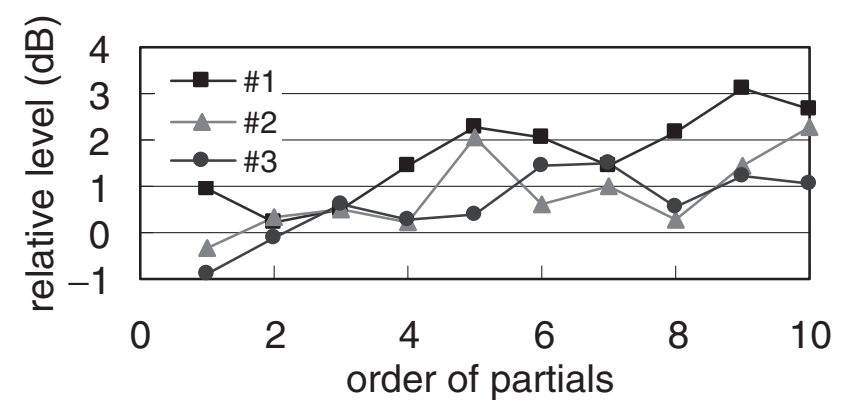

Fig. 8 Relative level differences of partials of pairs \#1, \#2, and \#3.

above, sound pairs made from a single original sound were used so that only the spectrum distributions were different. This is good because effects of the finger-key or keykeybed noise, even if it exists, may be very small, or have a negligible effect on the results of the test because the spectrum difference is in the frequency range from the first partial $(786.6 \mathrm{~Hz})$ and above. Now, the question arises whether two sounds recorded as a pair sound different or not. Three G5 pairs, namely \#1, \#2, and \#3, were chosen out of 8 pairs. The relative level differences of partials are shown in Fig. 8. A representation of level differences is similar to Fig. 4, but there is no level adjustment. The levels of partials of the hard touch sound are all larger than those of the soft touch in case of the pair \#1. It is the same for the pair \#2 except for the first partial and the same for the pair \#3 except for the first and the second partials. The fundamental component dominates the total energy of the G5 sound as seen in Fig. 2. Even though the hard touch sounds of \#2 and \#3 pairs have lower fundamental components (and therefore total energy levels) than the soft touch sounds, their partials have higher levels than the soft touch sounds. This indicates that the hard touch produces higher spectrum levels for higher partials if the striking strength is kept within some tolerance. It should be noted that, even though the peak levels monitored by a sound level meter were kept within $\pm 0.3 \mathrm{~dB}$, the actual level differences exceeded this limitation.

Only two subjects (D and I in Fig. 6) took part in the second stage listening tests simply because of the time limitation. Twenty pairs of hard touch sound (h) and soft touch sound (s) (ten (h)-(s) and ten (s)-(h) combinations) were presented to each subject. This series of tests was repeated three times, with each test conducted on different days. The results of the percentage of the correct judgments are shown in Table 2. It can be said that both subjects were able to discriminate the tone quality differences for the pairs \#1 and \#2. However, in the case of \#3, subject I has difficulty in discerning the tone quality. Subject D still can discern the sound (h) from the sound (s). As Fig. 8 shows, for pair \#1, the sound (h) has larger spectrum than the sound (s) for all partials, making the judgment easy for 
Table 2 Percentages of correct judgments of subjects D and I.

\begin{tabular}{cccccccc}
\hline & \multicolumn{3}{c}{$\mathrm{D}$} & & \multicolumn{3}{c}{$\mathrm{I}$} \\
\cline { 2 - 5 } \cline { 6 - 8 } & 1st & 2nd & 3rd & & 1st & 2nd & 3rd \\
\hline$\# 1$ & 90 & 95 & 95 & & 95 & 95 & 90 \\
$\# 2$ & 90 & 95 & 95 & & 85 & 90 & 95 \\
$\# 3$ & 80 & 70 & 80 & & 45 & 50 & 55 \\
\hline
\end{tabular}

both subjects. In the case of \#2, even though the level of the first partial of the sound (h) is smaller than that of the sound (s), the higher levels of partials of the sound (h) above the second partial compensate for the weakness of the fundamental. However, in the case of \#3, the level decrease of the first partial of the sound (h) is close to $1 \mathrm{~dB}$, making it difficult for subject I to judge that the sound (h) has larger higher order partials.

The above results show that not all, but at least, some percentage of listeners can hear the sound quality difference due to the touch. It would be safe to say that at least $10 \%$ of the people, who do not have specific training in music, can hear the difference in the case of G5.

The subjects were always asked about impressions on the sound quality difference. A common impression they expressed is that the sound (a) of the experiment in section 3.1 or the hard touch sound of the experiment in section 3.2 sounds "brighter" than its counterpart.

\section{CONCLUSIONS}

Physical and psychological experiments were conducted with an intention to determine whether piano tone quality is affected by the touch or not. The "hard" and "soft" touches were adopted because of the ease in producing the maximum tone quality differences by the difference of the touch. One of the important points in this study was the recording of piano sounds with the smallest possible intensity differences. Peak levels of recorded sounds were monitored so that the peak level difference in each comparison pair was within $\pm 0.3 \mathrm{~dB}$.

Ten sound sample pairs of notes G3, G4, and G5 were recorded and were analyzed after editing. Power spectrum differences between sounds of each pair were obtained and compared. It was found that, in cases of G3 and G4, the power spectrum differences due to the touch were negligible. However, in the case of G5, a trend was observed that the level of the partial of the hard touch sound becomes larger than that of the soft touch as the order of the partial increases. It seems that the level differences due to the touch are much less than pianists generally expect. However, it is also true that the touch can produce physical (spectrum) differences of sounds in some key ranges.

Listening tests conducted using G5 sounds showed that two piano sounds with artificially added spectrum level differences, which are equivalent to those produced by the hard and soft touches, were discernible to some percentage of the listeners.

The present study supports the pianist's opinion that the piano tone quality is affected by the touch. However, it is in a much lesser degree than most pianists expect. This study also indicates that, in the hammer-string interaction, the hammer must be considered (modeled) as a more complex system including the shank than the simple mass-spring system.

\section{ACKNOWLEDGMENTS}

The author would like to thank Mr. Jun Kanno and Ms. Sumiko Mikimoto for their cooperation in recording of the piano tones and useful discussions on the touch and the tone quality. The author is also very grateful to the students of author's laboratory who conducted the analysis and listening tests of the recorded sounds. The quality of this paper would have been greatly reduced without Mr. Charles Wurst's help to improve English expressions.

\section{REFERENCES}

[1] W. B. White, "Human elements in piano tone production," $J$. Acoust. Soc. Am., 1, 357-367 (1930).

[2] H. Hart, M. Fuller and W. Lusby, "A precision study of piano touch and tone," J. Acoust. Soc. Am., 6, 80-94 (1934).

[3] S. Yoshikawa, Does the Touch Change the Piano Timbre? The Physics in Musical Instruments- (Nikkei Science Inc., Tokyo, 1997).

[4] H. Suzuki, "Vibration analysis of a hammer-shank system," Interim Report of CBS Technology Center (1983).

[5] H. Suzuki, "Vibration analysis of a hammer-shank system," Proc. ISMA 1992, pp. 107-110 (1992).

[6] A. Askenfelt and E. V. Jansson, "From touch to string vibrations. II The motion of the key and the hammer," $J$. Acoust. Soc. Am., 90, 2383-2393 (1991).

[7] G. W. Koornhof and A. J. van der Walt, "The influence of touch on piano sound," Proc. Stockholm Music Acoustics Conf. (SMAC '93), No. 79, pp. 318-324 (1993).

[8] H. Miura, "The connection between technique and piano tone quality," Proc. Spring Meet. Acoust. Soc. Jpn., pp. 657-658 (1994).

[9] D. Naganuma and I. Nakamura, "Piano touch and analysis of tone quality," Proc. Spring Meet. Acoust. Soc. Jpn., pp. 657658 (1996).

[10] W. Goebl, R. Bresin and A. Galembo, "Once again: The perception of piano touch and tone. Can touch audibly change piano sound independently of intensity?," Proc. ISMA 2004, 4-S2-3, Nara, Japan (2004).

[11] H. Suzuki, J. Kanno and S. Mikimoto, "Analysis of piano tones with soft and hard touches," Proc. SMAC-03 (2003). 